\title{
Rate Distortion Theorem and the Multicritical Point of Spin Glass
}

\author{
Tatsuto Murayama* \\ Graduate School of Science and Engineering, \\ University of Toyama, 3190 Gofuku, \\ Toyama-shi, Toyama 930-8555, Japan \\ Asaki Saito ${ }^{\dagger}$ \\ Department of Complex and Intelligent Systems, \\ Future University Hakodate, 116-2 Kamedanakano-cho, \\ Hakodate, Hokkaido 041-8655, Japan \\ Peter Davis $\ddagger$ \\ Telecognix Corporation, 58-13 Yoshida Shimooji-cho, \\ Sakyo-ku, Kyoto-shi, Kyoto 606-8314, Japan
}

(Dated: August 27, 2020) 


\begin{abstract}
A spin system can be thought of as an information coding system that transfers information of the interaction configuration into information of the equilibrium state of the spin variables. Hence it can be expected that the relations between the interaction configuration and equilibrium states are consistent with the known laws of information theory. We show that Shannon's rate-distortion theorem can be used to obtain an universal constraint on neighboring spin correlations for a broad range of Ising spin systems with two-body spin interactions. Remarkably, this constraint gives a bound for the multicritical point in the phase diagram, when a mean-field behavior for the neighboring spin pairs can be expected in the paramagnetic phase.
\end{abstract}

Understanding the experimental observations of disordered materials has been a challenge to theoretical physicists. This triggered the rise of a special area of statistical mechanics that deals with a variety of statistical models with frozen disorder, where a series of mathematical techniques has become a common language for the systematic analysis [1,2]. Moreover, these techniques of statistical mechanics have been applied to the study of communication and information systems [3, 4], including noisy channel coding [5-7], recursive data compression [8-10], CDMA multiuser detection [11-13], modern cryptography [14], and some combinatorial optimization problems and methods for them [15-17]. Overall, the physicist's toolbox has successfully been applied to solve issues of information science; but not vice versa. To our knowledge, no classical theorem in information theory has been used to analyze the physics of complex condensed matter such as spin glass.

This Rapid Communication shows that information theory can be effectively applied to the analysis of spin glass systems. In our scenario, each of the equilibrium states of the Ising spins is regarded as one encoding of the interaction configuration [18, 19]. This scenario enables us to apply the Shannon rate-distortion theorem of information coding theory [20], which then allows us to develop a new method for investigating fundamental restrictions on the phase diagram. As a result, we obtain a previously unknown general bound for the location of the multicritical point for Ising spin glasses, where paramagnetic, ferromagnetic and spin glass phases merge [21]. Remarkably, our argument is independent of detail structure of the lattice. Numerical studies of problems related to the location of the

\footnotetext{
* murayama@eng.u-toyama.ac.jp

$\dagger$ saito@fun.ac.jp

$\ddagger$ davis@telecognix.com
} 
multicritical point for specific lattice models have been carried out by many physicists [22, 23]. However, we still have little knowledge about these significant issues from a theoretical point of view $[24,25]$.

In our spin glass model, we assign a binary spin $S_{i}= \pm 1$ to each site $i$ and the local energy $-J_{i j} S_{i} S_{j}$ to a set of pairwise bonds $(i, j)$ with a binary interaction $J_{i j}= \pm 1$. We investigate a class of Ising spin systems with the Hamiltonian

$$
\mathscr{H}\{\mathcal{S}\}\{\mathcal{J}\}=-\sum_{(i, j)} J_{i j} S_{i} S_{j}
$$

only assuming that the total number of the sites $i$ and the bonds $(i, j)$ are $N$ and $M$, respectively. Specifically, we do not restrict the range of the sum $(i, j)$ in (1). This sum could be over nearest neighbors, or it might include farther pairs, etc. Special features of each lattice will be reflected only through the ratio $R=N / M$. For the simplicity, this work deals with a Hamiltonian with two-body interactions to elucidate the benefit of Shannon's rate-distortion theorem, although the same arguments apply to other multi-body spin systems.

Each $J_{i j}$ is supposed to be distributed independently according to the common distribution

$$
P\left(J_{i j}\right)=p \delta\left(1, J_{i j}\right)+(1-p) \delta\left(-1, J_{i j}\right)
$$

for a value of disorder parameter $p$ in the interval $1 / 2<p \leq 1$. Here $\delta$ denotes the Kronecker's delta function and the set of interaction coefficients $\mathcal{J}=\left\{J_{i j}\right\}$ is called the $\operatorname{Bernoulli}(p)$ random variables. In general, we write the inverse temperature as $\beta$ and then the phase diagram of the system can be depicted in the space of disorder parameter $p$ and temperature $1 / \beta$. Now, we consider the Nishimori temperature $1 / \beta_{p}$ for the spin system, defined to be

$$
e^{2 \beta_{p}}=\frac{p}{1-p}
$$

Notice that the above equation specifies a line, the Nishimori line, in the space of $p$ and $1 / \beta[26]$. It has been shown that the multicritical point can be always found on this line. And so, we can specify the multicritical point by giving a value for the disorder parameter $p$, say $p_{c}$. Moreover, since spin glass phase does not exist on the Nishimori line, the multicritical point can be characterized as a ferromagnetic transition along the line [27]. 
In this Rapid Communication, we present a general bound for the location of the multicritical point of spin systems on any lattice with a Hamiltonian (1). Solid line in FIG. 1 shows the upper bound $p^{*}$ of $p_{c}$ for a given $R$, only below which we find the multicritical point. Notice that we can use Shannon's rate-distortion theorem to obtain this remarkable constraint when a mean-field behavior can be expected in the paramagnetic phase. More precisely, on the Nishimori line, we assume that

$$
P\left(S_{i}, S_{j} \mid \mathcal{J}\right) \simeq \exp \left(\beta_{p} J_{i j} S_{i} S_{j}\right)
$$

holds in the paramagnetic phase, where the $P\left(S_{i}, S_{j} \mid \mathcal{J}\right)$ denotes the joint distribution of $S_{i}$ and $S_{j}$ in the whole complex system and the $\simeq$ means equality up to a normalization constant. This implies that the effect of the rest of the lattice on local marginals should not be dominant and our potential target systems have a certain mean-field property in the paramagnetic state, at least on the Nishimori line. However, we insist that no further physical assumption is required to the Ising spin system. As an example, the dashed line in FIG. 1 represents the exact value $p_{c}$ of the multicritical point for a family of spin glass on a Bethe lattice [25]. Here all the bonds $(i, j)$ are chosen randomly to give a diluted lattice with the fixed connectivity of $2 / R$. The standard cavity analysis shows that the relation (2) holds at any temperature in the paramagnetic phase [26, 28]. As is expected, we can confirm that $p^{*}$ upper bounds $p_{c}$ for this specific model.

In the remainder of the work we will explain how this general bound can be obtained using the rate-distortion theorem. For the reader's convenience, we now outline the proof and then go into specific details afterwards. We first define an average of local correlation functions

$$
u=\left[\left\langle\frac{1}{M} \sum_{(i, j)} S_{i} S_{j}\right\rangle_{\beta}\right],
$$

where we assume that $\langle\cdot\rangle_{\beta}$ represents the expectation value in the equilibrium state of the Hamiltonian (1) at temperature $1 / \beta$, and suppose that a bracket $[\cdot]$ indicates averaging over an ensemble of configurations $\mathcal{J}$. If the assumption (2) holds within the paramagnetic phase, we always get $u=(2 p-1)^{2}$ at $1 / \beta_{p}$ for all $p<p_{c}$. However, if $R$ is small enough, $u=(2 p-1)^{2}$ derived from the paramagnetic assumption is smaller than the lower bound $u^{*}(p)$, which is imposed by Shannon's rate-distortion theorem. This implies that such $p$ for a given $R$ indicates the ferromagnetic state; otherwise contradiction. The infimum $p^{*}$ of 


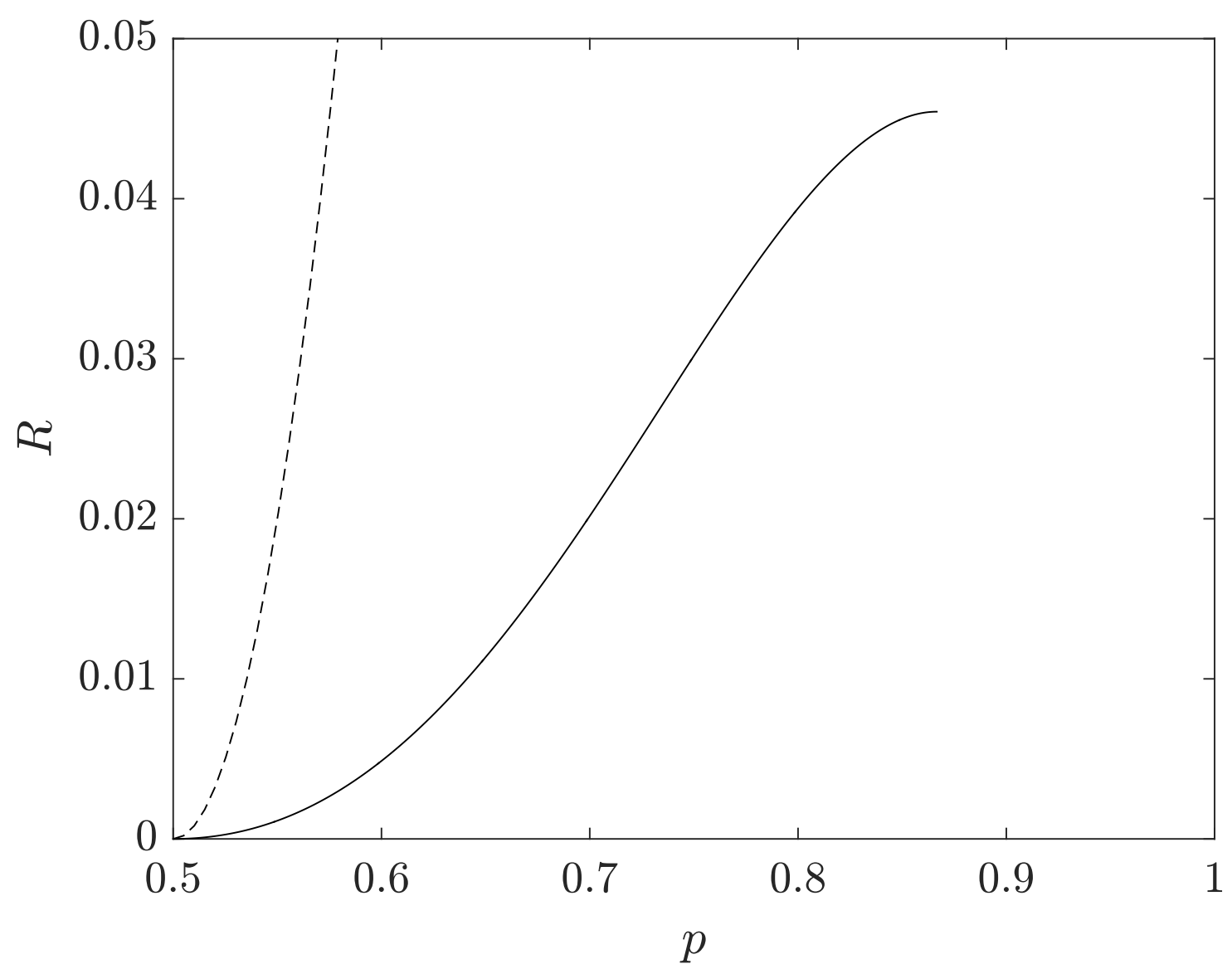

FIG. 1. Theoretical bound for the multicritical point in the phase diagram for disorder parameter $p$ and ratio $R=N / M$. The solid line represents the upper bound $p^{*}$ for the transition point $p_{c}$ for a given $R$. Shannon's rate-distortion theorem gives the constraint for $R$ smaller than 0.0454 . The dashed line represents the exact value of the transition point $p_{c}$ for a family of the Bethe lattice spin glass.

such ferromagnetic $p$, therefore, gives an upper bound for the transition point $p_{c}$. FIG. 2 illustrates a typical example with ratio $R=0.03$.

We first show that the local correlation function at $1 / \beta_{p}$ is

$$
u=(2 p-1)^{2},
$$

if the system is in the paramagnetic state. Since the relation (2) implies the explicit form

$$
P\left(S_{i}, S_{j} \mid \mathcal{J}\right)=\frac{1}{4 \cosh \beta_{p}} \exp \left(\beta_{p} J_{i j} S_{i} S_{j}\right)
$$


it is an easy matter to check that $\left\langle S_{i} S_{j}\right\rangle_{\beta_{p}}=\tanh \left(\beta_{p} J_{i j}\right)$ and averaging over $\mathcal{J}=\left\{J_{i j}\right\}$ gives $u=\left[\tanh \left(\beta_{p} J_{i j}\right)\right]=(2 p-1) \tanh \beta_{p}=(2 p-1)^{2}$.

Now, we will explain how to obtain theoretical bound $u^{*}(p)$ for $u$ based on Shannon's rate-distortion theorem. Let us first consider a virtual communication channel where the interaction configuration sequence $\mathcal{J}=\left\{J_{i j}\right\}$ is a set of the $\operatorname{Bernoulli}(p)$ random variables to be compressed, the set of spins $\mathcal{S}=\left\{S_{i}\right\}$ is its compressed representation/codeword, and the spin products $\hat{\mathcal{J}}=\left\{S_{i} S_{j}\right\}$ are its reproduction at Nishimori temperature $1 / \beta_{p}$. This choice of communication channel is motivated by the fact that at the Nishimori temperature, the Hamming distortion, or the normalized Hamming distance, $D=(1 / M) \sum_{(i, j)}\left[\left\langle\delta\left(-1, J_{i j} S_{i} S_{j}\right)\right\rangle_{\beta_{p}}\right]$ between the $\mathcal{J}$ and its reproduction $\hat{\mathcal{J}}$ can be easily obtained as $D=1-p[27]$. This specific distortion measure defines the goodness of $\hat{\mathcal{J}}$ as a representation of a set of given $\operatorname{Bernoulli}(p)$ random variables $\mathcal{J}$. The basic problem in Shannon's rate-distortion theory can then be stated as follows. What is the minimum description ratio $R=N / M$ required to achieve a given Hamming distortion D between the two sequences? Shannon's rate-distortion theorem gives the lower bound, say $R_{p}(D)$, as a function of the distortion measure $D$ for the theoretically achievable ratio $R=N / M$. The ratio, or rate, $R_{p}(D)$ is called the ratedistortion function for the $\operatorname{Bernoulli}(p)$ random variables. However, the distortion $D=1-p$ only gives a trivial lower bound $R_{p}(D)=R_{p}(1-p)=0$ and results in no restrictions for this specific channel [20].

We thus introduce a coding 'trick', a set of the Bernoulli $(\alpha)$ random variables $\tilde{\mathcal{J}}=\left\{\tilde{J}_{i j}\right\}$ with $1 / 2 \leq \alpha<p$, which allows us to tighten the bound on $R=N / M$. In the communication channel picture, the manipulation of the $\operatorname{Bernoulli}(\alpha)$ sequence $\tilde{\mathcal{J}}$ to get the sequence $\mathcal{J}$ corresponds to a preprocessing step in the encoding operation. After we preprocess $\tilde{\mathcal{J}}$ to get $\mathcal{J}$, the $\mathcal{J}$ is not Bernoulli $(p)$ assumed in the Nishimori's theory. However, this difference becomes negligible when we take the large system limit of $N \rightarrow \infty$. As a result, we can use the Nishimori's theory to calculate the Hamming distortion between $\tilde{\mathcal{J}}$ and $\hat{\mathcal{J}}$, which then offers a positive minimum ratio of $R=N / M$. Since distortion $D$ redefined for the new pair depends on $p$ and $u$, a positive bound on $R$ for the $D$, if any, imposes a constraint on $u$ as a function of $p$ and $R$. Hence, we obtain the theoretical lower bound $u^{*}(p)$ on $u$ for a given ratio $R=N / M$. Notice here that we require no physical assumptions such as (2) in this argument. In the following paragraphs we explain the essential details of this universal analysis. 
We first introduce a set $\tilde{\mathcal{J}}$ of $\operatorname{Bernoulli}(\alpha)$ random variables for some $\alpha$ satisfying $1 / 2 \leq$ $\alpha<p$. Define the set $T_{a}$ of all configurations with relative frequency of 1 s equal to $a$. For sufficiently large $M$, we can consider $\tilde{\mathcal{J}} \in T_{\alpha}$ and $\mathcal{J} \in T_{p}$, respectively [29]. So we suppose that any $\tilde{\mathcal{J}}$ configuration can be switched to a $\mathcal{J}$ configuration by flipping $(p-\alpha) M$ elements from -1 to 1 . We consider the set of spin products $\hat{\mathcal{J}}$ as an estimate of the original $\tilde{\mathcal{J}}$.

Here we evaluate the normalized Hamming distance between the samples $\tilde{\mathcal{J}}$ and $\hat{\mathcal{J}}$, i.e., $(1 / M) \sum_{(i, j)} \delta\left(-1, \tilde{J}_{i j} S_{i} S_{j}\right)$. We first notice that the identity $\tilde{J}_{i j} S_{i} S_{j}=J_{i j} \tilde{J}_{i j} \cdot J_{i j} S_{i} S_{j}$ leads to

$$
\sum_{(i, j)} \delta\left(-1, \tilde{J}_{i j} S_{i} S_{j}\right) \leq \sum_{(i, j)} \delta\left(-1, J_{i j} \tilde{J}_{i j}\right)+\sum_{(i, j)} \delta\left(-1, J_{i j} S_{i} S_{j}\right)
$$

The equality holds if and only if there is no chance of getting $J_{i j} \tilde{J}_{i j}=-1$ and $J_{i j} S_{i} S_{j}=-1$ simultaneously. By definition, the preprocessing gives $\sum_{(i, j)} \delta\left(-1, J_{i j} \tilde{J}_{i j}\right)=(p-\alpha) M$. The second term on the right would be

$$
\left[\left\langle\sum_{(i, j)} \delta\left(-1, J_{i j} S_{i} S_{j}\right)\right\rangle_{\beta_{p}}\right]=(1-p) M
$$

since the gauge theory tells us that the internal energy becomes $\left[\langle\mathscr{H}\{\mathcal{S}\}\{\mathcal{J}\}\rangle_{\beta_{p}}\right]=$ $-M \tanh \beta_{p}$ on the Nishimori line [26]. Assume that the bracket [ $\left.\cdot\right]$ also indicates averaging over an ensemble of configurations $\tilde{\mathcal{J}}$ as well as $\mathcal{J}$. Then we have

$$
\left[\left\langle\sum_{(i, j)} \delta\left(-1, \tilde{J}_{i j} S_{i} S_{j}\right)\right\rangle_{\beta_{p}}\right] \leq(1-\alpha) M
$$

To directly calculate the Hamming distance between the samples $\tilde{\mathcal{J}}$ and $\hat{\mathcal{J}}$ on the Nishimori line, we introduce a pair of auxiliary variables $Q_{1 \rightarrow-1}$ and $Q_{-1 \rightarrow 1}$ defined to be

$$
\begin{aligned}
Q_{-1 \rightarrow 1}(1-p) M+Q_{1 \rightarrow-1} p M & =(1-p) M, \\
\left(1-Q_{-1 \rightarrow 1}\right)(1-p) M+Q_{1 \rightarrow-1} p M & =(1-q) M,
\end{aligned}
$$

where $Q_{x \rightarrow y}$ is the empirical probability of $S_{i} S_{j}=y$ when $J_{i j}=x$ and $q$ denotes a frequency of 1 s at the random variables $\hat{\mathcal{J}}$. Notice that the former equation just counts up every difference $J_{i j} \neq S_{i} S_{j}$, while the latter indicates the total number of $S_{i} S_{j}=-1$ in the reconstruction. By solving the two equations, we have

$$
Q_{1 \rightarrow-1}=\frac{1-q}{2 p}, \quad Q_{-1 \rightarrow 1}=1-\frac{1-q}{2(1-p)} .
$$


It is easy to check that these formulas are well defined as probabilities in the interval $2 p-1 \leq$ $q \leq 1$ for a given $p \neq 1$. Notice also that $u=2 q-1$. Then it follows that

$$
\left[\left\langle\sum_{(i, j)} \delta\left(-1, \tilde{J}_{i j} S_{i} S_{j}\right)\right\rangle_{\beta_{p}}\right]=(1-\alpha) M-2 Q_{1 \rightarrow-1}(p-\alpha) M
$$

(see [26]). In other words, the normalized Hamming distance between $\tilde{\mathcal{J}}$ and $\hat{\mathcal{J}}$ on the Nishimori line can be estimated by the formula

$$
d_{\alpha}(p, q)=(1-\alpha)-2 Q_{1 \rightarrow-1}(p-\alpha)
$$

which is non-negative for the relevant intervals.

Lastly, it is possible to invoke Shannon's rate-distortion theorem for the Bernoulli $(\alpha)$ random variables [20]. In the new communication channel picture with preprocessing, we first write $D=(1 / M) \sum_{(i, j)}\left[\left\langle\delta\left(-1, \tilde{J}_{i j} S_{i} S_{j}\right)\right\rangle_{\beta_{p}}\right]$ and focus on the Hamming distortion between the original $\tilde{\mathcal{J}}$ and its reproduction $\hat{\mathcal{J}}$. Define the rate-distortion function for the $\operatorname{Bernoulli}(\alpha)$ random variables as

$$
R_{\alpha}(D)=H_{2}(\alpha)-H_{2}(D)
$$

where we denote $H_{2}(\alpha)=-\alpha \log _{2}(\alpha)-(1-\alpha) \log _{2}(1-\alpha)$. For the ratio $R=N / M$ and the distortion $D$, the theorem states that

$$
R_{\alpha}(D)<R
$$

This inequality provides a bound on the compression ratio $R$, dependent only on distortion $D$. By letting $D=d_{\alpha}(p, q)$, we can use the formula $R_{\alpha}(p, q)=H_{2}(\alpha)-H_{2}\left(d_{\alpha}(p, q)\right)$ to lower bound the ratio as $R_{\alpha}(p, q)<R$ for every $\alpha$ in the relevant interval $1 / 2 \leq \alpha<p$. Now write

$$
R^{*}(p, q)=\sup _{1 / 2 \leq \alpha<p} R_{\alpha}(p, q)
$$

It is obvious that we can still lower bound $R$ as

$$
R^{*}(p, q) \leq R
$$

Notice also that the $R^{*}(p, q)$ is a non-increasing continuous function of $q$. Suppose that the ratio $R=N / M$ is small enough to satisfy an inequality $R<R^{*}(p, 2 p-1)$. Here the $R^{*}(p, 2 p-1)$ is the largest value of $R^{*}(p, q)$ for $q$ over the interval $2 p-1 \leq q \leq 1$. Since 


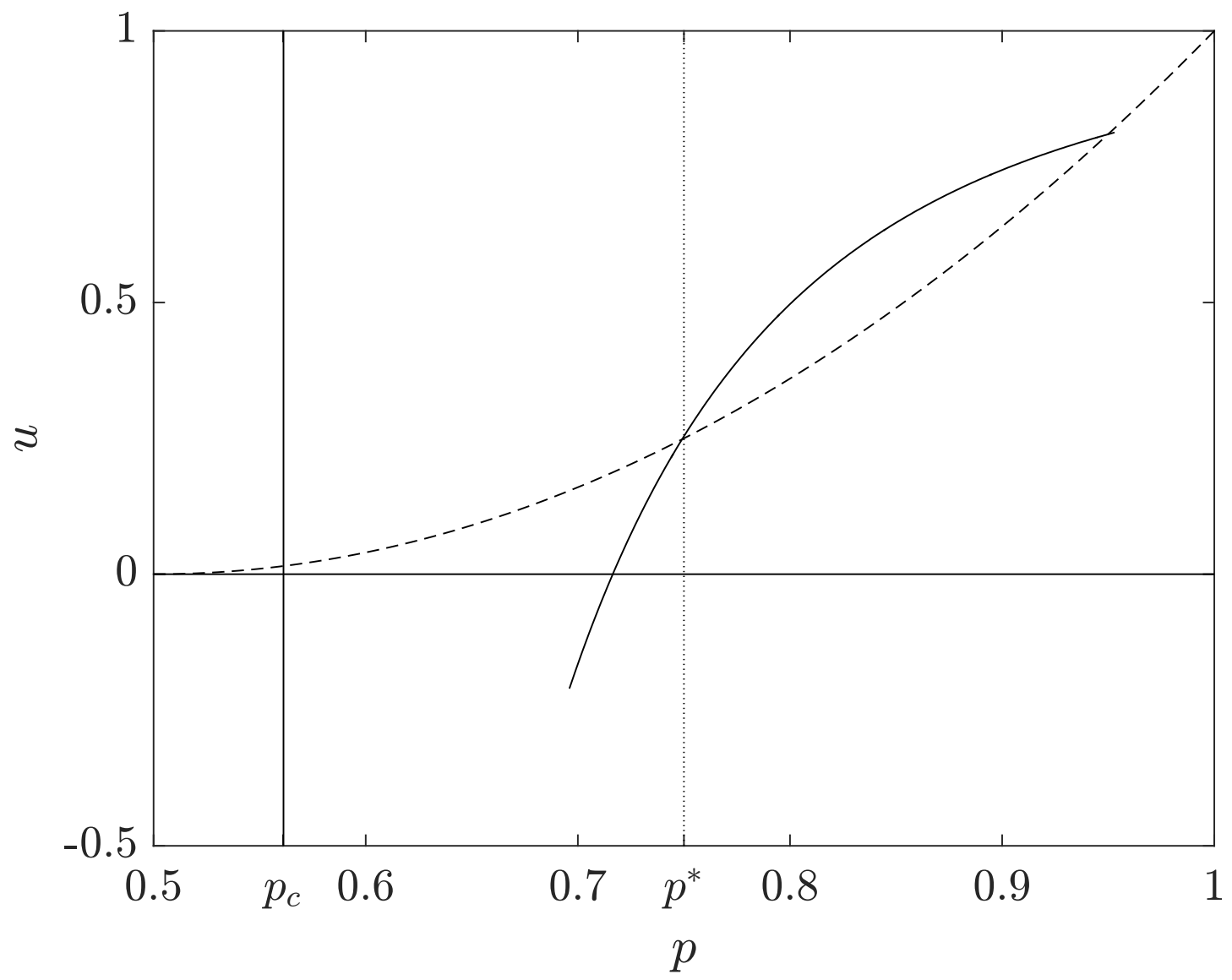

FIG. 2. Theoretical constraints for local spin product $u$ as a function of disorder parameter $p$ for ratio $R=0.03$. The solid curve represents the universal lower bound of $u$ imposed by Shannon's rate-distortion theorem. The dashed parabolic curve indicates the calculation of $u$ based on a mean-field assumption for neighboring spin marginals. The paramagnetic solution contradicts our universal lower bound for $p$ greater than the intersection point $p^{*}=0.750$. This means that for $p>p^{*}$ the paramagnetic solution is no more the stable solution, implying that $p_{c} \leq p^{*}$. The vertical solid line shows $p_{c}=0.561$ for a family of the Bethe lattice spin glass.

$d_{\alpha}(p, 1)=1-\alpha$, it is an easy matter to check that $R^{*}(p, 1)=0$ for every $p$. Then, by the intermediate value theorem, there exists a number $q^{*}(p)$ in the closed interval $2 p-1 \leq q \leq 1$ such that

$$
R^{*}\left(p, q^{*}(p)\right)=R
$$


We compare the formulas (6) and (7) to conclude that

$$
q^{*}(p) \leq q,
$$

i.e., the $q^{*}(p)$ lower bounds $q$.

For the $R=N / M$ small enough, we numerically examine the equation (7) which implicitly determines $q^{*}(p)$ for a given pair of $p$ and $R$. Evaluation of the equation $R^{*}(p, 2 p-1)=R$ shows that there exists such a solution $q^{*}(p)$ for some $p$ for every $R$ smaller than 0.0541 . Notice that the lower bound $q^{*}(p)$ for the Bernoulli parameter $q$ gives the lower bound $u^{*}(p)=2 q^{*}(p)-1$ for local spin product $u$. FIG. 2 compares this universal lower bound $u^{*}(p)$ with the preceding paramagnetic solution $u=(2 p-1)^{2}$. However, in this figure, $u=(2 p-1)^{2}$ violates our lower bound $u^{*}(p)$ for $p$ larger than the intersection point $p^{*}$. Hence, the $p$ larger than $p^{*}$ implies the ferromagnetic phase, in which the paramagnetic solution could break down. In other words, the multicritical transition point $p_{c}$ should be smaller than the intersection point $p^{*}$. For a given $R$, this $p^{*}$ offers an upper bound for $p_{c}$ as is shown by the solid line in FIG. 1, which is identified with $R^{*}\left(p, 2 p^{2}-2 p+1\right)=R$.

In this Rapid Communication, we considered the ' $N$-bit' spin state of the Ising spin glass model as compressed representations of a set of $M$ Bernoulli $(p)$ binary random variables encoded in the interaction configuration. We showed that the Shannon rate-distortion theorem, which provides a bound on the compression ratio dependent only on distortion, can give an upper bound $p^{*}$ for the location of the multicritical point $p_{c}$ for a sufficiently small compression ratio $R=N / M$. Remarkably, our argument is independent of detail structure of the lattice and only requires a mean-field assumption for the joint marginals of neighboring spins in the paramagnetic phase. Results obtained here for a certain class of lattice models with two-body Ising spin interactions will motivate applications of Shannon's rate-distortion theorem to other Ising spin systems.

\section{Acknowledgments}

We would like to thank Federico Ricci-Tersenghi and Yoshiyuki Kabashima for useful discussions. We also thank an anonymous reviewer for suggestions that improved the explanations. This work was in part supported by JSPS KAKENHI Grant Numbers JP16KK0005, 
JP17K00009.

[1] M. Mézard, G. Parisi, and M. A. Virasoro, Spin Glass Theory and Beyond (World Scientific, 1987).

[2] M. Mézard and A. Montanari, Information, Physics, and Computation (Oxford University Press, 2009).

[3] T. Richardson and R. Urbanke, Modern Coding Theory (Cambridge University Press, 2008).

[4] N. Merhav, Foundations and Trends® in Communications and Information Theory 6, 1 (2010).

[5] N. Sourlas, Nature 339, 693 (1989).

[6] R. Vicente, D. Saad, and Y. Kabashima, Physical Review E 60, 5352 (1999).

[7] N. Macris, IEEE Transactions on Information Theory 53, 664 (2007).

[8] T. Murayama, Physical Review E 69, 035105 (2004).

[9] S. Ciliberti, M. Mézard, and R. Zecchina, Physical Review Letters 95, 038701 (2005).

[10] M. J. Wainwright, E. Maneva, and E. Martinian, IEEE Transactions on Information Theory 56, 135 (2010).

[11] T. Tanaka, IEEE Transactions on Information theory 48, 2888 (2002).

[12] D. Guo and S. Verdú, IEEE Transactions on Information Theory 51, 1983 (2005).

[13] K. Mimura and M. Okada, IEEE Transactions on Information Theory 60, 3645 (2014).

[14] Y. Kabashima, T. Murayama, and D. Saad, Physical Review Letters 84, 2030 (2000).

[15] K. Y. M. Wong and D. Sherrington, Journal of Physics A: Mathematical and General 20, L793 (1987).

[16] K. Hukushima and K. Nemoto, Journal of the Physical Society of Japan 65, 1604 (1996).

[17] R. Monasson, R. Zecchina, S. Kirkpatrick, B. Selman, and L. Troyansky, Nature 400, 133 (1999).

[18] M. Opper and D. Saad, Advanced Mean Field Methods: Theory and Practice (MIT press, 2001).

[19] M. Talagrand, Spin Glasses: A Challenge for Mathematicians: Cavity and Mean Field Models (Springer, 2003).

[20] T. M. Cover and J. A. Thomas, Elements of Information Theory (John Wiley \& Sons, 2012). 
[21] H. Nishimori and D. Sherrington, AIP Conference Proceedings 553, 67 (2001).

[22] P. L. Doussal and A. B. Harris, Physical Review Letters 61, 625 (1988).

[23] R. R. P. Singh, Physical Review Letters 67, 899 (1991).

[24] A. Honecker, M. Picco, and P. Pujol, Physical Review Letters 87, 047201 (2001).

[25] G. Parisi, F. Ricci-Tersenghi, and T. Rizzo, Journal of Statistical Mechanics: Theory and Experiment 2014, P04013 (2014).

[26] See Supplemental Material for some remarks and detailed calculations.

[27] H. Nishimori, Statistical Physics of Spin Glasses and Information Processing: An Introduction (Oxford University Press, 2001).

[28] M. Mézard and G. Parisi, The European Physical Journal B-Condensed Matter and Complex Systems 20, 217 (2001).

[29] I. Csiszar and J. Körner, Information theory: coding theorems for discrete memoryless systems (Cambridge University Press, 2011). 


\title{
Supplemental Material: Rate Distortion Theorem and the Multicritical Point of Spin Glass
}

\author{
Tatsuto Murayama* \\ Graduate School of Science and Engineering, \\ University of Toyama, 3190 Gofuku, \\ Toyama-shi, Toyama 930-8555, Japan \\ Asaki Saito ${ }^{\dagger}$ \\ Department of Complex and Intelligent Systems, \\ Future University Hakodate, 116-2 Kamedanakano-cho, \\ Hakodate, Hokkaido 041-8655, Japan \\ Peter Davis $^{\ddagger}$ \\ Telecognix Corporation, 58-13 Yoshida Shimooji-cho, \\ Sakyo-ku, Kyoto-shi, Kyoto 606-8314, Japan
}

(Dated: August 27, 2020) 
In this brief document, we give some supportive information to help the readers grasp background knowledge and understand technical details about our Rapid Communication. Notice that a number for each formula in this document is identical to the number we have already assigned in the associated work.

Remarks on the Nishimori Line. We first illustrate a schematic picture for the location of the multicritical point, Nishimori line, and the three phase boundaries in the space of $p$ and $T=1 / \beta$. As is dipicted in FIG. 1 the ferromagnetic phase transition along the Nishimori line coincides with the multicritical point, which enables us to identify the critical value $p_{c}$ for $p$ in the phase diagram.

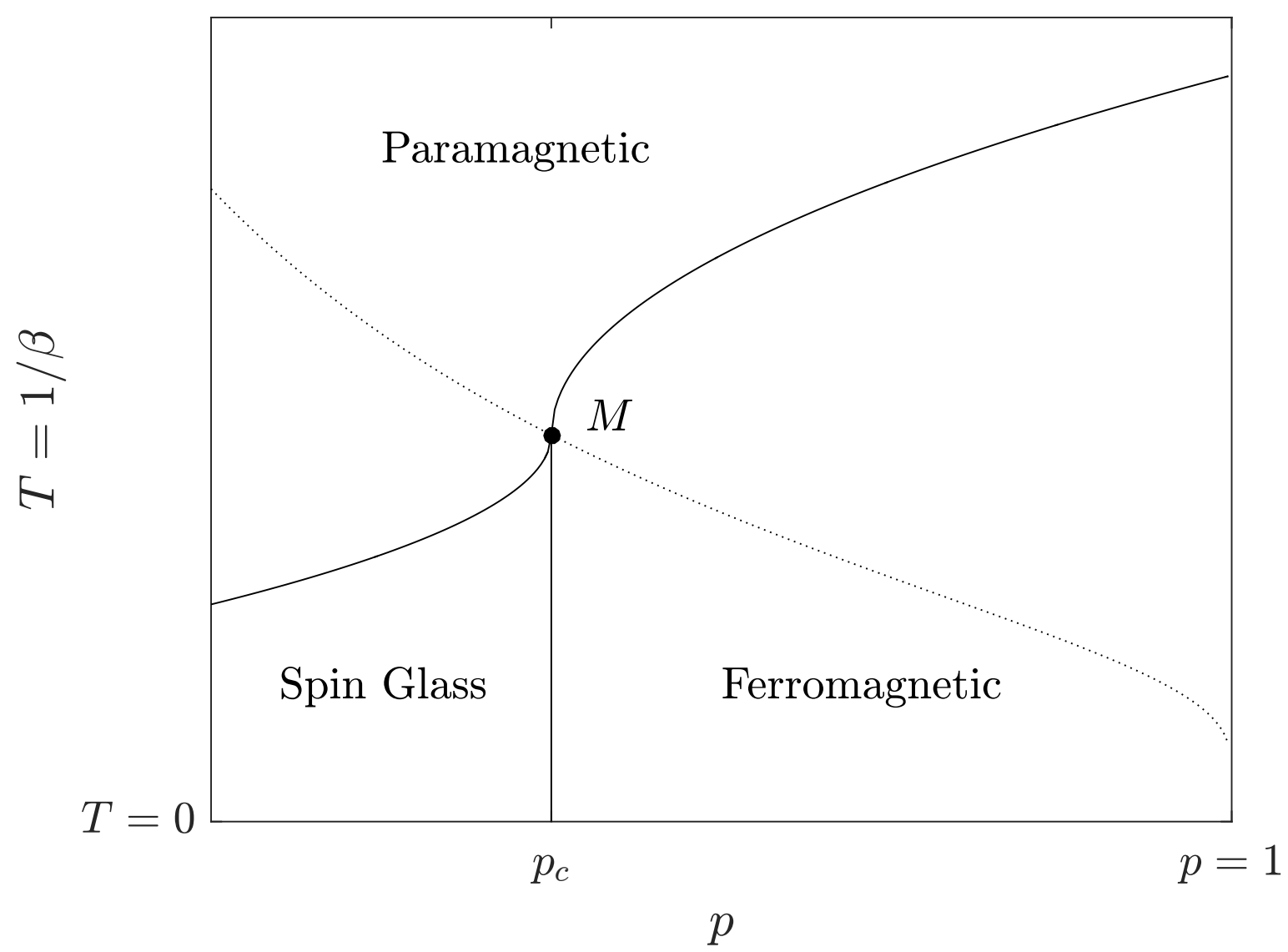

FIG. 1. A schematic illustration of Nishimori line in the Ising spin glass models. The $M$ denotes the location of the multicritical point on the Nishimori line, where paramagnetic, ferromagnetic, and spin glass phases merge. 
Remarks on the Bethe Lattice. We show that the Bethe lattice spin glass models satisfy the relation (2), providing a pedagogical example. We first notice that the Hamiltonian (1) gives

$$
\mathscr{H}\{\mathcal{S}\}\{\mathcal{J}\}=-\sum_{(i, j)} J_{i j} S_{i} S_{j}=-\sum_{j \in \partial i} J_{i j} S_{i} S_{j}+\widehat{\mathscr{H}}_{i}
$$

The $\partial i$ represents a collection of all sites $j$ connected to $i$ with an interaction $J_{i j}$. The $\widehat{\mathscr{H}}_{i}$ denotes the Hamiltonian of a smaller Ising spin system without the spin variable $S_{i}$. Notice that the cavity field $h_{j \rightarrow i}$ is well defined on the Bethe lattice through the equation

$$
\widehat{\mathscr{H}_{i}}=-\sum_{j \in \partial i} h_{j \rightarrow i} S_{j}
$$

Then the effective Hamiltonian for the site $i$ and its vicinity is found to be

$$
\widehat{\mathscr{H}}=-\sum_{j \in \partial i} J_{i j} S_{i} S_{j}-\sum_{j \in \partial i} h_{j \rightarrow i} S_{j}
$$

It follows that

$$
\begin{aligned}
P\left(S_{i} \mid \mathcal{J}\right) & =\frac{1}{\mathcal{Z}_{1}} \sum_{\left\{S_{j}\right\}_{j \in \partial i}} \exp (-\beta \widehat{\mathscr{H}}) \\
& =\frac{1}{\mathcal{Z}_{1}} \sum_{\left\{S_{j}\right\}_{j \in \partial i}} \prod_{j \in \partial i} \exp \left(\beta J_{i j} S_{i} S_{j}+\beta h_{j \rightarrow i} S_{j}\right)=\frac{1}{\mathcal{Z}_{1}} \prod_{j \in \partial i} \sum_{S_{j}= \pm 1} \exp \left(\beta J_{i j} S_{i} S_{j}+\beta h_{j \rightarrow i} S_{j}\right) .
\end{aligned}
$$

Here $\mathcal{Z}_{1}$ denotes the normalization constant. Since $S_{j}= \pm 1$, the identity

$$
\exp \left\{\beta S_{j}\left(J_{i j} S_{i}+h_{j \rightarrow i}\right)\right\}=2 \cosh \left\{\beta\left(J_{i j} S_{i}+h_{j \rightarrow i}\right)\right\} \frac{1+S_{j} \tanh \left\{\beta\left(J_{i j} S_{i}+h_{j \rightarrow i}\right)\right\}}{2}
$$

gives

$$
\frac{1}{\mathcal{Z}_{1}} \prod_{j \in \partial i} \sum_{S_{j}= \pm 1} \exp \left(\beta J_{i j} S_{i} S_{j}+\beta h_{j \rightarrow i} S_{j}\right)=\frac{1}{\mathcal{Z}_{1}} \prod_{j \in \partial i}\left\{2 \cosh \left\{\beta\left(J_{i j} S_{i}+h_{j \rightarrow i}\right)\right\}\right\} .
$$

Now we define the effective field

$$
\hat{h}_{j \rightarrow i}=\frac{1}{\beta} \tanh ^{-1}\left\{\tanh \left(\beta J_{i j}\right) \tanh \left(\beta h_{j \rightarrow i}\right)\right\} .
$$

Together with another identity

$$
\cosh \left\{\beta\left(J_{i j} S_{i}+h_{j \rightarrow i}\right)\right\}=2 \cosh \left(\beta J_{i j}\right) \cosh \left(\beta h_{j \rightarrow i}\right) \frac{1+S_{i} \tanh \left(\beta J_{i j}\right) \tanh \left(\beta h_{j \rightarrow i}\right)}{2}
$$


we have

$$
P\left(S_{i} \mid \mathcal{J}\right)=\frac{1}{\mathcal{Z}_{2}} \exp \left(\beta S_{i} \sum_{j \in \partial i} \hat{h}_{j \rightarrow i}\right)
$$

with a new normalization constant $\mathcal{Z}_{2}$. Since the magnetization at site $i$ is

$$
m_{i}=\frac{1}{\mathcal{Z}_{2}} \sum_{S_{i}= \pm 1} S_{i} \exp \left(\beta S_{i} \sum_{j \in \partial i} \hat{h}_{j \rightarrow i}\right)
$$

it is an easy matter to check that $h_{j \rightarrow i}=0$ for all $j$ gives $m_{i}=0$. By letting $h_{j \rightarrow i}=0$, the summation over the spin variables other than $S_{i}$ and $S_{j}$ results in

$$
P\left(S_{i}, S_{j} \mid \mathcal{J}\right)=\frac{1}{\mathcal{Z}_{3}} \sum_{\left\{S_{k}\right\}_{k \in \partial i \backslash j}} \exp \left(\beta \sum_{k \in \partial i} J_{i k} S_{i} S_{k}\right)
$$

where $\mathcal{Z}_{3}$ denotes the normalization constant of the joint marginal distribution. Finally, simple algebra gives

$$
P\left(S_{i}, S_{j} \mid \mathcal{J}\right)=\frac{1}{4 \cosh \beta} \exp \left(\beta J_{i j} S_{i} S_{j}\right)
$$

This indicates that the relation (2) holds at any temperature in the paramagnetic phase for the Bethe lattice spin glass models and, hence, the location of their multicritical points in the phase diagram should be consistent with our results.

Derivation of Equation (3). First, the gauge theory tells us that the internal energy becomes

$$
\frac{-1}{M}\left[\langle\mathscr{H}\{\mathcal{S}\}\{\mathcal{J}\}\rangle_{\beta_{p}}\right]=\tanh \beta_{p}=2 p-1
$$

at the Nishimori temperature $1 / \beta_{p}$. Notice that the above formula holds for any lattice. Together with the definition of the Hamiltonian (1), i.e.,

$$
\mathscr{H}\{\mathcal{S}\}\{\mathcal{J}\}=-\sum_{(i, j)} J_{i j} S_{i} S_{j}=-\sum_{(i, j)} \delta\left(1, J_{i j} S_{i} S_{j}\right)+\sum_{(i, j)} \delta\left(-1, J_{i j} S_{i} S_{j}\right)
$$

we get

$$
\left[\left\langle\sum_{(i, j)} \delta\left(1, J_{i j} S_{i} S_{j}\right)\right\rangle_{\beta_{p}}\right]-\left[\left\langle\left\langle\sum_{(i, j)} \delta\left(-1, J_{i j} S_{i} S_{j}\right)\right\rangle_{\beta_{p}}\right]=(2 p-1) M .\right.
$$

Notice also that

$$
\left[\left\langle\sum_{(i, j)} \delta\left(1, J_{i j} S_{i} S_{j}\right)\right\rangle_{\beta_{p}}\right]+\left[\left\langle\sum_{(i, j)} \delta\left(-1, J_{i j} S_{i} S_{j}\right)\right\rangle_{\beta_{p}}\right]=M
$$


holds. Then the conclusion follows, i.e.,

$$
\left[\left\langle\sum_{(i, j)} \delta\left(-1, J_{i j} S_{i} S_{j}\right)\right\rangle_{\beta_{p}}\right]=(1-p) M
$$

Derivation of Equation (5). We put

$$
\begin{aligned}
& C=\left\{(i, j) \mid \tilde{J}_{i j}=-1 \text { or } 1\right\} \\
& D=\left\{(i, j) \mid \tilde{J}_{i j}=-1, J_{i j}=1, S_{i} S_{j}=-1\right\} .
\end{aligned}
$$

Obviously, we have

$$
\sum_{(i, j) \in D} \delta\left(-1, \tilde{J}_{i j} S_{i} S_{j}\right)=0, \quad \sum_{(i, j) \in D} \delta\left(-1, J_{i j} \tilde{J}_{i j}\right)=|D|, \quad \sum_{(i, j) \in D} \delta\left(-1, J_{i j} S_{i} S_{j}\right)=|D|
$$

where $|D|$ is the number of the elements in $D$. As we discussed in the derivation of equation (4), we have

$$
\sum_{(i, j) \in C \backslash D} \delta\left(-1, \tilde{J}_{i j} S_{i} S_{j}\right)=\sum_{(i, j) \in C \backslash D} \delta\left(-1, J_{i j} \tilde{J}_{i j}\right)+\sum_{(i, j) \in C \backslash D} \delta\left(-1, J_{i j} S_{i} S_{j}\right) .
$$

Thus, we have

$$
\begin{aligned}
\sum_{(i, j) \in C} \delta\left(-1, \tilde{J}_{i j} S_{i} S_{j}\right) & =\sum_{(i, j) \in C \backslash D} \delta\left(-1, \tilde{J}_{i j} S_{i} S_{j}\right)+\sum_{(i, j) \in D} \delta\left(-1, \tilde{J}_{i j} S_{i} S_{j}\right) \\
& =\sum_{(i, j) \in C \backslash D} \delta\left(-1, J_{i j} \tilde{J}_{i j}\right)+\sum_{(i, j) \in C \backslash D} \delta\left(-1, J_{i j} S_{i} S_{j}\right) \\
& =\sum_{(i, j) \in C} \delta\left(-1, J_{i j} \tilde{J}_{i j}\right)+\sum_{(i, j) \in C} \delta\left(-1, J_{i j} S_{i} S_{j}\right)-2|D|
\end{aligned}
$$

Taking the average, we obtain

$$
\left[\left\langle\sum_{(i, j)} \delta\left(-1, \tilde{J}_{i j} S_{i} S_{j}\right)\right\rangle_{\beta_{p}}\right]=(1-\alpha) M-2 Q_{1 \rightarrow-1}(p-\alpha) M
$$

\footnotetext{
* murayama@eng.u-toyama.ac.jp

$\dagger$ saito@fun.ac.jp

$\ddagger$ davis@telecognix.com
} 